\title{
Characterization and Growth Mechanism of Nickel Nanowires Resulting from Reduction of Nickel Formate in Polyol Medium
}

\author{
Olga A. Logutenko, Alexander I. Titkov, Alexander M. Vorob'yov, \\ Yriy M. Yukhin, and Nikolay Z. Lyakhov \\ Institute of Solid State Chemistry and Mechanochemistry, Siberian Branch of the Russian Academy of Sciences, \\ Kutateladze 18, Novosibirsk 630128, Russia \\ Correspondence should be addressed to Olga A. Logutenko; ologutenko@solid.nsc.ru
}

Received 29 August 2016; Revised 2 November 2016; Accepted 13 November 2016

Academic Editor: Jean M. Greneche

Copyright (C) 2016 Olga A. Logutenko et al. This is an open access article distributed under the Creative Commons Attribution License, which permits unrestricted use, distribution, and reproduction in any medium, provided the original work is properly cited.

\begin{abstract}
Nickel linear nanostructures were synthesized by reduction of nickel formate with hydrazine hydrate in ethylene glycol medium in the absence of any surfactants or capping agents for direction of the particles growth. The effect of the synthesis conditions such as temperature, reduction time, type of polyol, and nickel formate concentration on the reduction products was studied. The size and morphology of the nickel nanowires were characterized by X-ray diffraction, scanning, and transmission electron microscopy. It was shown that the nickel nanocrystallites were wire-shaped with a face-center-cubic phase. Ethylene glycol was found to play a crucial role in the formation of the nickel nanowires. The possible growth processes of the wire-shaped particles taking place at 110 and $130^{\circ} \mathrm{C}$ are discussed. It was shown that, under certain synthesis conditions, nickel nanowires grow on the surface of the crystals of the solid intermediate of nickel with hydrazine hydrate.
\end{abstract}

\section{Introduction}

In recent years, transition metal nanoparticles (Fe, Co, and $\mathrm{Ni}$ ) have received much attention owing to their wide applications in catalysis, optical, electronic, and magnetic devices $[1,2]$. There are many various techniques that are used for making metal nanoparticles with different morphologies such as spherical nanoparticles [3], triangles [4], nanorods [5], chains [2], and nanowires [6-11]. Anisotropic magnetic nanomaterials are known to exhibit unique magnetic properties due to which one-dimensional (1D) nanostructured materials have great potential for use in optical, electronic, and magnetic devices [12,13]. As to 1D metallic nickel, it is increasingly required for use in gas sensors, magnetic recording devices, catalysts, and drug deliveries [14-16]. The magnetic properties of nickel nanoparticles allow their application in cell separation and manipulation. Thus, nickel nanowires functionalized with antibodies can be used for efficient cell separation [17] with little or no effects on cells and no disruption to the cellular growth cycle [18].

To date, a range of $1 \mathrm{D}$ structures have been synthesized for various transition metals including nickel. To prepare 1D nanostructures, such as nanorods, nanofibers, and nanowires, the template assisted method spatially restricting the particle growth $[19,20]$ or the magnetic-field assisted growth route in which magnetic particles align under a magnetic field forming linear chains [21-24] is often used in practice. Thus, polycrystalline nickel nanowires of around 10 microns in length and of about $200 \mathrm{~nm}$ in diameter were obtained using magnetic induction [25]. However, these methods which have drawbacks are being quite complicated which is caused by multistep preparation of the matrix for synthesis and the subsequent purification of the final product from residues and contaminants. Furthermore, they do not allow production of a large amount of nanomaterials.

The chemical reduction route for synthesis of nanoparticles is the most widely used because it is relatively simple, allows control of the size and the size distribution of the particles, and is more promising in terms of industrial implementation due to its relative simplicity and low cost. In this case, to restrict radial growth of nanowires, various surfactants were noted to be suitable instead of the conventional templates. The surfactants, acting as capping agents, can change the ratio 
of the growth rates of different crystallographic planes of the particles and result in the formation of one-dimensional nanostructures. Generally, PVP is used as the surfactant which can direct the growth of wire-like structures and prevent them from aggregation $[2,11]$. Apart from that, the formation of nanorods was shown to be promoted by a high hexadecylamine content in the reaction medium [26]. Liu et al. [27] found that the anion surfactant sodium dodecyl benzenesulfonate played a key role in the growth of $\mathrm{Ni}$ nanobelts.

However, some experimental results indicated that the capping material is not always essential for the growth of the Ni nanowires. Thus, Krishnadas et al. [7] synthesized nickel nanowires via the reduction of nickel chloride in ethylene glycol using hydrazine hydrate as the reducing agent without the assistance of surfactants, templates, and external magnetic field. They showed that an increase in the nickel concentration resulted in an increase in both the diameter (from 60 to $130 \mathrm{~nm}$ ) and the length (from $\sim 1.50$ to $\sim 6.00 \mu \mathrm{m}$ ) of the nanowires. They also found that temperature plays an important role in determining the dimensions and morphology of the nanowires, while the surfactant and magnetic field have negligible roles in their formation. However, despite the authors providing some details into the growth of anisotropic nickel nanostructures, the exact mechanism of their formation, including the role of the solvent in this process, remained unclear. Also, the effect of the nature of the solvent on the formation of the nanowires was not considered by the authors.

In this paper, we present the results of a study of the reduction of nickel formate with hydrazine hydrate using polyol (ethylene glycol, diethylene glycol, 1,2-propylene glycol, and 1,5-pentanediol) as a medium and provide a fairly simple method for preparation of nickel nanowires having the potential for high-volume production. No morphologycontrolling media, such as external magnetic forces, template materials, and surfactants, were needed to promote the one-dimensional growth in this system. Nickel formate was chosen as a precursor because the possibility of the use of short chain carboxylates for stabilization of nickel nanoparticles has not been studied yet. Furthermore, unlike long chain carboxylates, nickel formate is quite soluble in ethylene glycol, which allows carrying out the reduction of the precursor in the solution. Also, washing the final product (powder metal) from impurities is much easier in this case. The effect of the synthesis conditions (temperature, reduction time, nickel formate concentration, and the nature of the polyol) on the nanowires growth process has been studied and a tentative mechanism of their formation is discussed.

\section{Experimental}

Nickel (II) formate dihydrate $\left(\mathrm{Ni}(\mathrm{HCOO})_{2} \cdot 2 \mathrm{H}_{2} \mathrm{O}\right)$ and ethylene glycol $\left(\mathrm{HO}\left(\mathrm{CH}_{2}\right)_{2} \mathrm{OH}\right)$ of $99.8 \%$ purity grade, $\geq 99.5 \%$ pure 1,2-propylene glycol $\left(\mathrm{CH}_{3} \mathrm{CH}(\mathrm{OH}) \mathrm{CH}_{2} \mathrm{OH}\right), \geq 97.0 \%$ pure 1,5-pentanediol $\left(\mathrm{HO}\left(\mathrm{CH}_{2}\right)_{5} \mathrm{OH}\right), 99 \%$ pure diethylene glycol $\left(\left(\mathrm{HOCH}_{2} \mathrm{CH}_{2}\right)_{2} \mathrm{O}\right)$, 95\% pure ethanol, and $80 \%$ solution of hydrazine hydrate supplied by Sigma Aldrich were used in the experiments without further purification. Solutions were prepared using distilled water.

The reduction of nickel formate was carried out as follows. An appropriate amount of nickel formate was dissolved in a polyol under stirring by heating in an oil bath to the desired temperature. Then a concentrated solution $(80 \%)$ of hydrazine hydrate was added to the reaction mixture under continuous mechanical stirring at a nickel to hydrazine hydrate molar ratio of $1: 18$. After the reaction was complete, the mixture was air-cooled and the supernate was decanted. The resulting nickel powder was washed several times with ethanol and distilled water to remove the impurities and then it was dried in air at room temperature. The particles size and their morphology were investigated by means of X-ray diffraction (XRD) analysis and electron microscopy.

XRD patterns were recorded on a D8 Advance powder $\mathrm{X}$-ray diffractometer equipped with a one-dimensional LynxEye detector and a $\mathrm{K}_{\beta}$ filter using $\mathrm{Cu} \mathrm{K} \alpha$ radiation. The crystallite size and lattice parameters were estimated by the Rietveld method [28] using software for the profile and structural analysis Topas 4.2 (Bruker AXS, Germany). The broadening of the patterns due to the crystallite size was modeled by the "Double-Voigt" function. Analysis of the samples by transmission electron microscopy (TEM) was performed using a JEM 2010 electron microscope (JEOL, Japan) operating at $200 \mathrm{kV}$ and having a resolution of $0.14 \mathrm{~nm}$. Study of the samples by scanning electron microscopy (SEM) was performed using a Hitachi $3400 \mathrm{~N}$ scanning electron microscope (Hitachi Ltd., Japan).

The carbon, hydrogen, and nitrogen contents in the synthesized samples were determined by the modified PreglDumas method ending in gravimetric analysis using a Perkin Elmer 2400 Elemental Analyzer. The nickel content in the solution was determined by complexometric titration in the presence of the indicator murexide.

\section{Results and Discussion}

Ethylene glycol (EG) can act as a reducing agent as well as the reaction medium in many systems. However, in the case of nickel, its reduction to metal requires the process to be carried out in the presence of alkaline agents and at the boiling point of ethylene glycol $\left(\sim 198^{\circ} \mathrm{C}\right)$ [29] which makes the control of size and morphology very difficult. To reduce nickel at lower temperatures, additional reducing agents need to be introduced into the system. In our work we used hydrazine hydrate for that. To accelerate the reduction reaction rate, the solution should be alkaline enough as the reduction of nickel ions and generation of nanoparticles require the synthesis to be carried out at $\mathrm{pH}$ values of $>9$. In the case of nickel formate, the reduction reaction proceeds without adding alkaline reagents to the system which excludes the formation of nickel hydroxide as an intermediate and avoids contamination of the final product by them.

It should be noted that ethylene glycol was also found to play the role of a protective layer on the particle surfaces preventing them from agglomerating which might occur via interactions between $\mathrm{OH}$ groups and nickel atoms [30, 31]. That is why we did not introduce additional stabilizing 


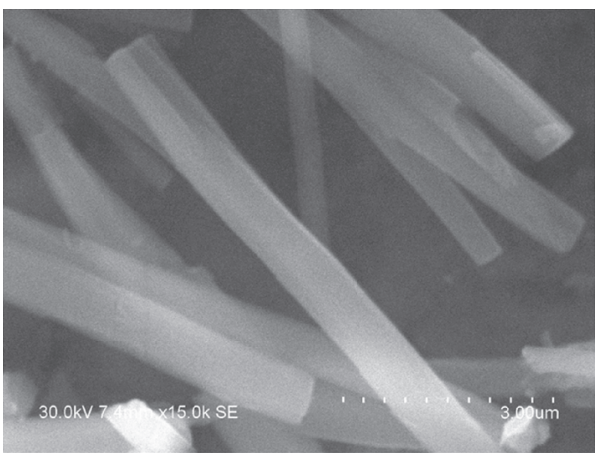

(a)

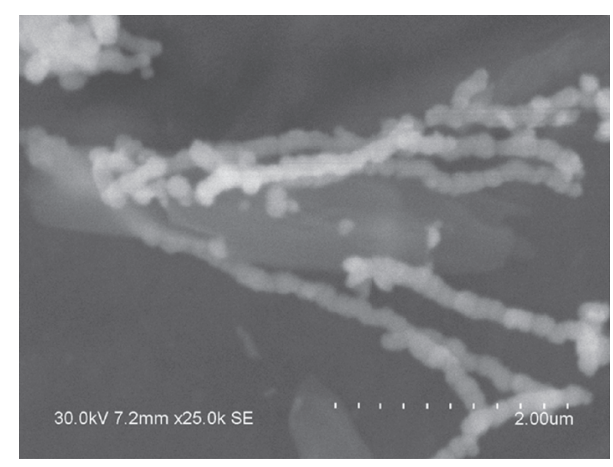

(b)

FIGURE 1: SEM images of the samples obtained by reduction of nickel formate with hydrazine hydrate at temperatures of $100^{\circ} \mathrm{C}(\mathrm{a})$ and $110^{\circ} \mathrm{C}$ (b).

reagents into the reaction medium. Lond et al. found [32] that, when dissolving a nickel chloride in ethylene glycol, a complex of the composition $\mathrm{NiCl}_{2}(\mathrm{EG})_{3}$ is formed.

\subsection{Effect of the Reduction Temperature and Time on the} Properties of the Nickel Nanoparticles. In order to determine the optimum temperature required for the reduction of nickel formate with hydrazine hydrate in ethylene glycol and to understand the influence of temperature, a series of experiments was carried out at reaction temperatures of $80,100,110,120,130$, and $150^{\circ} \mathrm{C}$ and a nickel formate to ethylene glycol weight ratio of $1: 100$. It was shown that the reaction rate increased as the temperature increased. Thus, at temperatures below $110^{\circ} \mathrm{C}$, the reduction does not occur even over a time interval of $4 \mathrm{~h}$. After adding hydrazine hydrate to the solution of nickel formate in ethylene glycol at $100^{\circ} \mathrm{C}$, a light violet-pink precipitate is formed. As follows from the X-ray diffraction (XRD) and scanning electron microscopy (SEM) analysis, the precipitate obtained under these conditions does not contain metallic nickel and has quite highly elongated crystal morphology. The crystals are uniform in size and have a length of about 7-9 microns and a width of about 300 to $600 \mathrm{~nm}$ (Figure $1(\mathrm{a})$ ). At $110^{\circ} \mathrm{C}$, according to the XRD data, the incomplete reduction of nickel formate occurs (Figure 2, curve 2). As seen from the figure, the product obtained is a mixture of metallic nickel (Bragg's reflections at $2 \Theta$ value of $44.5^{\circ}, 51.98^{\circ}$, and $76.4^{\circ}$ ) and some nickel compound precipitated first from the solution. This is also confirmed by the electron microscopy data (Figure 1(b)). As seen from the electron microscopy images, the final product contains large crystals of the unreacted nickel compound of $\sim 1.5 \mu \mathrm{m}$ in width and $\sim 8 \mu \mathrm{m}$ in length and small (100-150 $\mathrm{nm}$ in size) spherical nickel nanoparticles nucleated directly on the crystals from which the nanowires are growing.

At $120^{\circ} \mathrm{C}$, the reduction reaction proceeds slowly resulting in the formation of a mixture containing mostly metallic nickel with the impurity of the initial complex of nickel formate with hydrazine hydrate. At a temperature of $130^{\circ} \mathrm{C}$, when adding hydrazine hydrate to the hot solution of nickel formate in ethylene glycol, the reaction mixture changes from

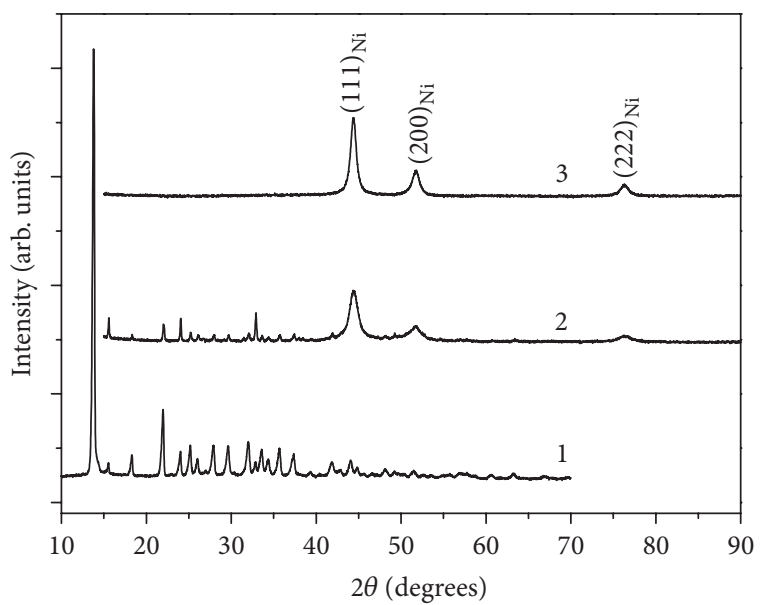

FIGURE 2: XRD patterns of the samples obtained by reduction of nickel formate with hydrazine hydrate at different reduction temperatures, ${ }^{\circ} \mathrm{C}: 100$ (1), 110 (2), and 130 (3).

a green colour to deep violet-blue but remains transparent due to the formation of some soluble nickel complex with hydrazine hydrate. For a while, no precipitate formation took place under these conditions. However, 1-2 minutes later, the solution becomes turbid and black, and solid product appears indicating the formation of metallic nickel. Figure 2 (curve 3) shows the XRD patterns of the precipitate obtained at $130^{\circ} \mathrm{C}$. As seen, only the diffraction peaks at $2 \theta$ values of $44.5^{\circ}, 51.98^{\circ}$, and $76,4^{\circ}$ attributed to the main characteristic peaks of the (111), (200), and (222) crystal planes of facecentered cubic (fcc) nickel (JCPDS file number 04-0850) are observed. There are no other crystalline phases, including $\mathrm{NiO}$, in the diffraction patterns which indicate that the pure metallic nickel could be obtained under these conditions. The lack of oxidation of the nickel nanoparticles prepared in ethylene glycol via the reduction by hydrazine hydrate has been discussed in many publications, for example, $[6,33]$, and was attributed to the formation of nitrogen gas during the reaction process which could prevent the nickel nanoparticles from oxidation. However, even though the characteristic 


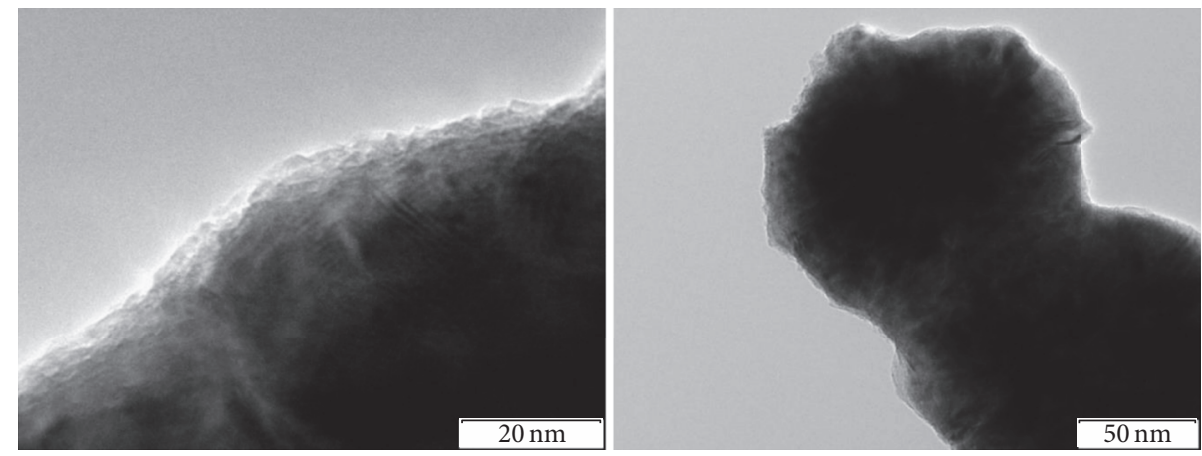

FIGURE 3: TEM images of nickel nanowires synthesized by reduction of nickel formate with hydrazine hydrate at $130^{\circ} \mathrm{C}$.

peaks assigned to the formation of nickel oxide were not detected in the XRD patterns (Figure 2, curve 3), analysis of the nickel nanoparticles obtained by transmission electron microscopy (Figure 3) revealed the presence of a very small layer of amorphous nickel oxide on the nanowires surface. The thickness of the layer does not exceed $1-2 \mathrm{~nm}$ and it does not increase over time. The morphology of the samples investigated by TEM and SEM showed (Figures 3, 4(a), and $4(\mathrm{~b})$ ) that the obtained particles are wire-like and nanocrystalline. Their average size is about $100 \mathrm{~nm}$ in diameter and their length ranges from 2 to 4 microns. According to XRD data, the crystallite size of the resulting nickel particles was $13 \mathrm{~nm}$. It should be noted that no individual spherical nickel particles are observed in the SEM image and the wires have a quite uniform shape.

An increase in the temperature up to 140 and $150^{\circ} \mathrm{C}$ results in an increase in the rate of the nickel reduction, in the first place, but does not change very much the dimensions and morphology of the resultant nanoparticles. The results of studies of the obtained powders by SEM showed that the particles have a wire-like shape and their diameter ranges from 150 to $200 \mathrm{~nm}$ while their length is in the range of 7$10 \mu \mathrm{m}$. It is also seen that the wires are less homogeneous in the resultant powders. According to XRD data, the products obtained are pure metallic nickel with a crystallite size of $9 \mathrm{~nm}$. Thus, at temperatures below $130^{\circ} \mathrm{C}$, the reduction process proceeds very slowly or it does not occur at all. An increase in temperature above $130^{\circ} \mathrm{C}$ results in an increase of both the diameter and the length of the nanowires but the increase in length is more noticeable than the increase in diameter. Apart from that, an increase in temperature results in a large variation in length so the temperature of $(130 \pm 5)^{\circ} \mathrm{C}$ seems optimal for the synthesis of nickel nanowires.

In order to investigate the effect of reaction time, nickel nanoparticles were synthesized at a reduction time of $1 \mathrm{~min}$ and 1 hour. SEM images of the samples obtained at different reaction times showed (Figures 4(c) and 4(d)) that, at the initial stage of reaction, in the first $1 \mathrm{~min}$, nickel nanowires are already properly formed which indicates that process of the nanowire formation is rapid. The resulting particles have a size of about $150 \mathrm{~nm}$ in width and $6-10 \mu \mathrm{m}$ in length. A further increase in the reaction time results in little change in the dimensions of the nanowires but makes them less uniform (Figure 4(d)). As seen from the figure, some nanowires become thicker due to the aggregation process, reaching approximately $300 \mathrm{~nm}$ in diameter, while their length remains almost the same. According to the XRD data, an increase in reduction time results in an increase in the crystallite size from 9 to $12 \mathrm{~nm}$.

\subsection{Effect of Nickel Formate Concentration on the Morphology} of the Nickel Nanoparticles. The effect of the nickel concentration in the initial solution on the size and morphology of the resulting nickel particles has been investigated at $130^{\circ} \mathrm{C}$ with fixed nickel to hydrazine hydrate molar ratio of $1: 18$ and reaction time of $3 \mathrm{~min}$. The concentrations of nickel in ethylene glycol varied from $0.005 \mathrm{~mol} / \mathrm{L}$ to $0.27 \mathrm{~mol} / \mathrm{L}$. In Figures 4(e) and 4(f), SEM micrographs of nickel nanowires prepared at nickel formate to ethylene glycol weight ratios of $1: 20(0.27 \mathrm{~mol} / \mathrm{L} \mathrm{Ni})$ and $1: 400(0.0135 \mathrm{~mol} / \mathrm{L} \mathrm{Ni})$ are shown. As seen from this figure, a decrease in the nickel formate concentration results in a decrease in the dimensions of the nanowires. At the same time, the distribution of the length of the nanowires becomes much broader, with predominant shorter chains, as the initial nickel concentration in the solution is decreased. Thus, average diameters of the nanowires are approximately $300,150,100$, and $50 \mathrm{~nm}$ at nickel to ethylene glycol ratios of $1: 20,1: 100,1: 400$, and $1: 1000$, respectively. Their lengths almost do not change remaining in the range of 2-3 $\mu \mathrm{m}$ when changing the nickel to ethylene glycol ratio from $1: 20$ to $1: 1000$ but the distribution of the length of the nickel nanowires tends to be wider with a decrease in nickel concentration. Therefore, the concentration of nickel in the reaction system should be kept in the $0.05-0.2 \mathrm{~mol} / \mathrm{L}$ range to retain the wire-like morphology of the synthesized nickel nanoparticles.

3.3. Mechanism of Nanowire Formation. The mechanism of 1D nanostructures growth is widely discussed in the literature $[7,27,34,35]$ but it has not yet been fully clarified not least because many factors affect the growth process. So the proposed mechanisms for nanowire formation are mostly qualitative and all authors who tried to precisely describe the growth process of the $1 \mathrm{D}$ nickel nanostructures concluded that it is a problem. In the case of our system, the possible reactions resulting in the formation of nickel nanowires can be presented as follows. Initially, when nickel formate is dissolved in ethylene glycol, the formation of the 


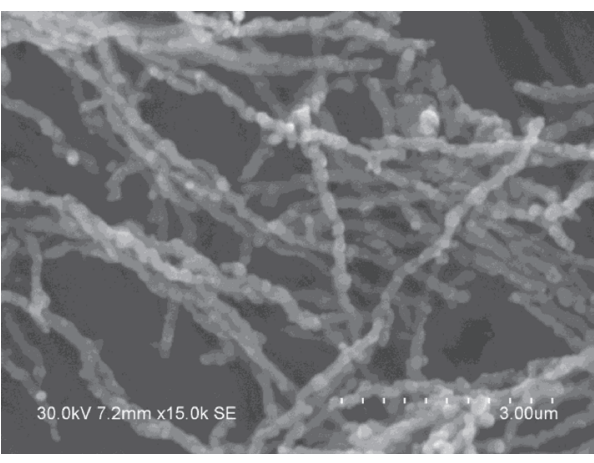

(a) $\mathrm{T}=130^{\circ} \mathrm{C}, \mathrm{Ni}: \mathrm{EG}=1: 100$, and $\tau=3 \mathrm{~min}$

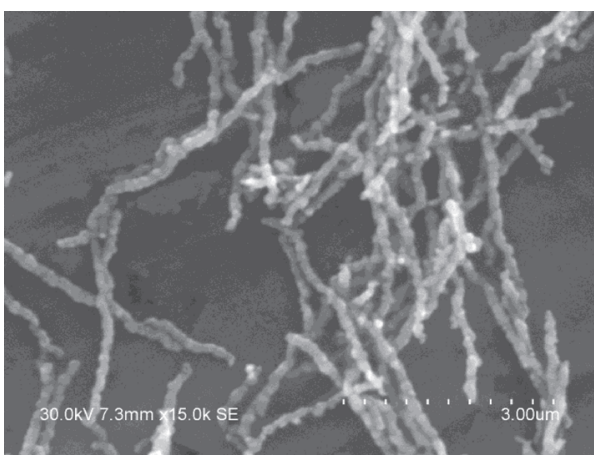

(c) $\tau=1 \mathrm{~min}, 130^{\circ} \mathrm{C}$, and $\mathrm{Ni}: \mathrm{EG}=1: 100$

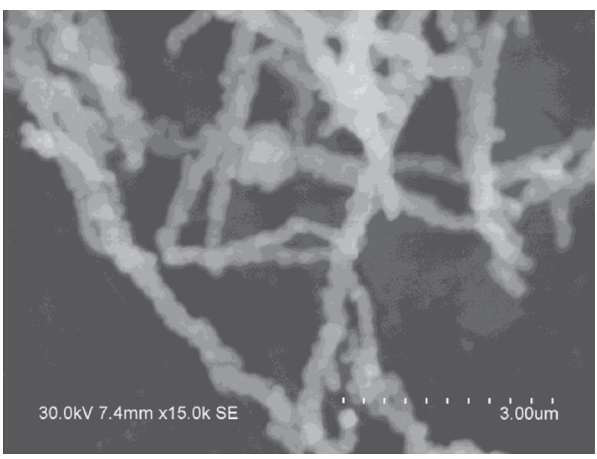

(e) $\mathrm{Ni}: \mathrm{EG}=1: 20,130^{\circ} \mathrm{C}$, and $\tau=3 \mathrm{~min}$

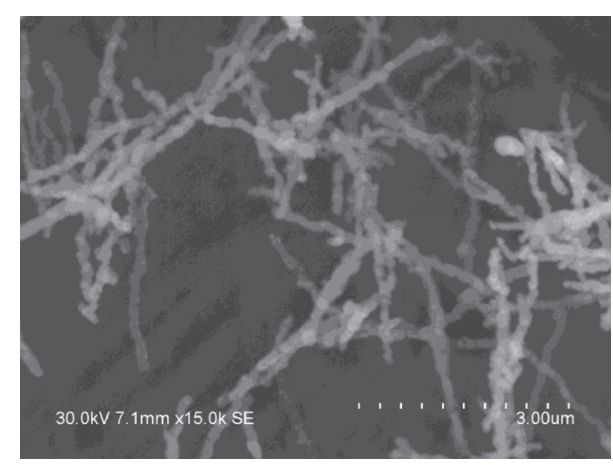

(b) $\mathrm{T}=140^{\circ} \mathrm{C}, \mathrm{Ni}: \mathrm{EG}=1: 100$, and $\tau=3 \mathrm{~min}$

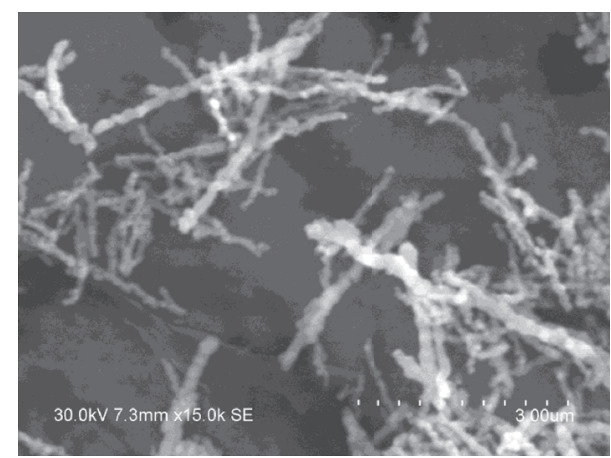

(d) $\tau=1 \mathrm{~h}, 130^{\circ} \mathrm{C}$, and $\mathrm{Ni}: \mathrm{EG}=1: 100$

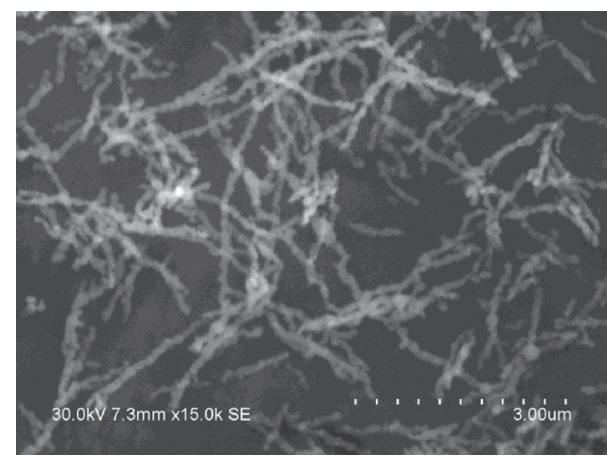

(f) $\mathrm{Ni}: \mathrm{EG}=1: 400,130^{\circ} \mathrm{C}$, and $\tau=3 \mathrm{~min}$

FIGURE 4: SEM images of nickel nanowires synthesized by reduction of nickel formate with hydrazine hydrate at different temperatures (a, b), reaction times (c, d), and nickel concentrations (e, f).

nickel formate species solvated by ethylene glycol molecules occurs (1). Then, when adding hydrazine hydrate to the solution, ethylene glycol ligands are exchanged for $\mathrm{N}_{2} \mathrm{H}_{4}$ ligands resulting in the formation of a more stable complex between nickel formate and hydrazine hydrate as described by (2). This complex is precipitated from the solution at temperatures below $130^{\circ} \mathrm{C}$ as a violet-pink compound, while at temperatures above $130^{\circ} \mathrm{C}$ it is soluble. The change in the solution colour from green to deep violet-blue confirms its formation. As known from the literature [36], hydrazine hydrate can play a role in both a bridging bidentate ligand towards the metal and the reduction agent for the metal. The complex composition was shown to depend on the amount of hydrazine hydrate in the system and on the acidity of the reaction medium. According to published information
[36-38], several complexes can be present in the solution containing nickel chloride and hydrazine hydrate, such as $\mathrm{Ni}\left(\mathrm{N}_{2} \mathrm{H}_{4}\right)_{2} \mathrm{Cl}_{2}, \mathrm{Ni}\left(\mathrm{N}_{2} \mathrm{H}_{4}\right)_{3} \mathrm{Cl}_{2}$, and $\mathrm{Ni}\left(\mathrm{N}_{2} \mathrm{H}_{4}\right)_{6} \mathrm{Cl}_{2}$, which act as precursors for the production of nickel nanoparticles.

$$
\begin{aligned}
& \mathrm{Ni}(\mathrm{HCOO})_{2} \cdot 2 \mathrm{H}_{2} \mathrm{O}+n \mathrm{HOCH}_{2} \mathrm{CH}_{2} \mathrm{OH} \\
& \quad \longrightarrow\left[\mathrm{Ni}\left(\mathrm{HOCH}_{2} \mathrm{CH}_{2} \mathrm{OH}\right)_{n}\right](\mathrm{HCOO})_{2}+2 \mathrm{H}_{2} \mathrm{O} \\
& \quad\left[\mathrm{Ni}\left(\mathrm{HOCH}_{2} \mathrm{CH}_{2} \mathrm{OH}\right)_{n}\right](\mathrm{HCOO})_{2}+m \mathrm{~N}_{2} \mathrm{H}_{4} \\
& \quad \longrightarrow\left[\mathrm{Ni}_{(}\left(\mathrm{N}_{2} \mathrm{H}_{4}\right)_{m}\right](\mathrm{HCOO})_{2}+n \mathrm{HOCH}_{2} \mathrm{CH}_{2} \mathrm{OH}
\end{aligned}
$$

As the solution is heated for longer, the free (excess) hydrazine reduces dissolved nickel species followed by the nucleation and growth of the nickel particles as chain-like 


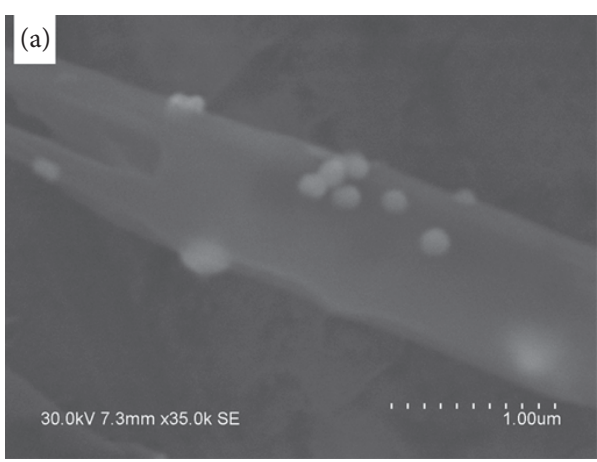

(a)

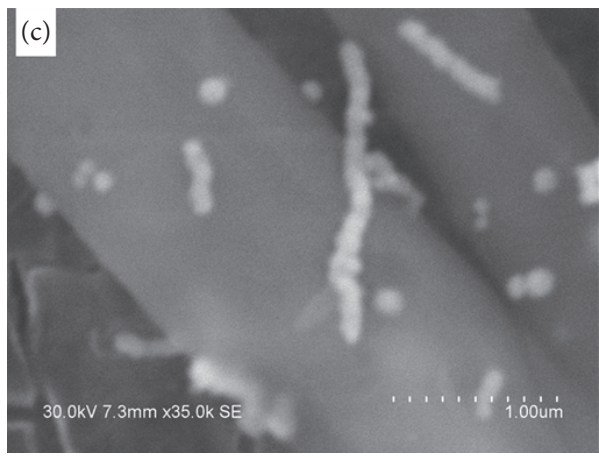

(c)

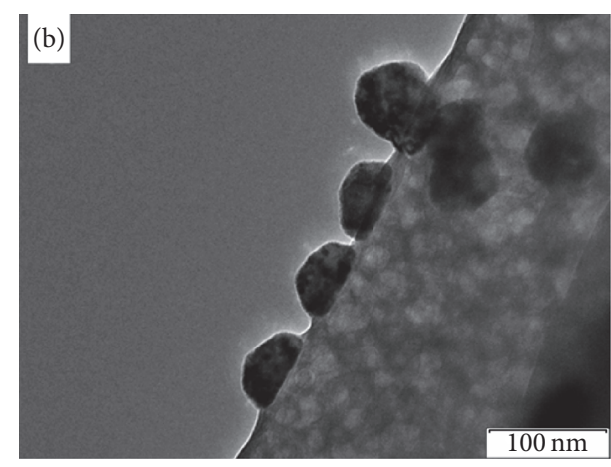

(b)

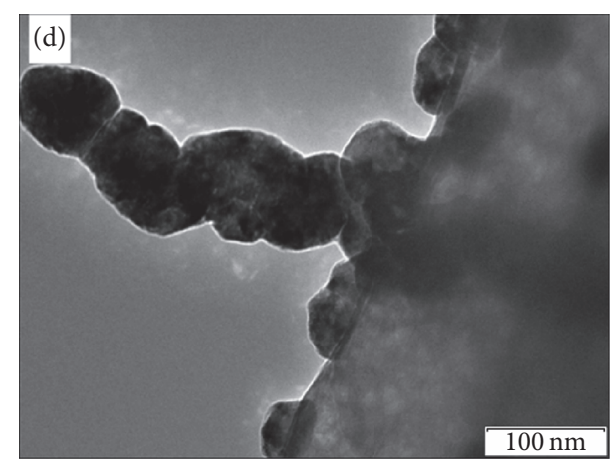

(d)

FIgURE 5: SEM (a, c) and TEM (b, d) images of the nanoparticles obtained by reduction of $\mathrm{Ni}_{(}\left(\mathrm{N}_{2} \mathrm{H}_{4}\right)_{2}(\mathrm{CHOO})_{2}$ in ethylene glycol with hydrazine hydrate after different reaction time periods at $130^{\circ} \mathrm{C}$. (a, b) $1 \mathrm{~min}$ and (c, d) $2 \mathrm{~min}$; $\mathrm{Ni}: \mathrm{EG}=1: 100$.

nanostructures via homogeneous nucleation (3). The reaction proceeds very quickly resulting in a very rapid change in colour from gray to black.

$$
\begin{gathered}
4\left[\mathrm{Ni}\left(\mathrm{N}_{2} \mathrm{H}_{4}\right)_{m}\right]^{2+}+4 n \mathrm{~N}_{2} \mathrm{H}_{4} \\
\longrightarrow 4 \mathrm{Ni}+4(m+n) \mathrm{NH}_{3}+2(m+n) \mathrm{N}_{2} \\
+(m+n) \mathrm{H}_{2}+2(m+n) \mathrm{H}^{+}
\end{gathered}
$$

Since, at temperatures below $130^{\circ} \mathrm{C}$, nickel nanowires grow from seeds initially formed on the crystals precipitated from the solution, we isolated the intermediate obtained by the addition of hydrazine hydrate to the solution of nickel formate in ethylene glycol at $100^{\circ} \mathrm{C}$, first studying its morphology and composition and then the process of its reduction in ethylene glycol with hydrazine hydrate. As mentioned above, at temperatures below $130^{\circ} \mathrm{C}\left(100-110^{\circ} \mathrm{C}\right)$, as soon as hydrazine hydrate was added to the solution of nickel formate, the solution immediately became turbid and a light violet-pink precipitate was formed. The precipitate so obtained was separated from the solution by centrifugation and washed with ethanol several times. Then, it was dried in air and sent for elemental composition analysis. Analysis of the samples revealed the presence of nickel, nitrogen, carbon, hydrogen, and oxygen. Based on the weight percentages of 27.0, 26.2, 4.5, 11.5, and 30.5 obtained for $\mathrm{Ni}, \mathrm{N}, \mathrm{H}, \mathrm{C}$, and $\mathrm{O}$, respectively, the empirical formula of the complex obtained was found to be $\mathrm{Ni}\left(\mathrm{N}_{2} \mathrm{H}_{4}\right)_{2}(\mathrm{CHOO})_{2}$. The calculated weight percentages for $\mathrm{Ni}, \mathrm{N}, \mathrm{H}, \mathrm{C}$, and $\mathrm{O}$ are 27,6;26.3; 4.7; 11,3; 30,1 respectively. It should be noted that the same composition was found for the compounds precipitated from ethylene glycol at $\mathrm{Ni}$ to ethylene glycol ratio of 1:20 and from the solution of propylene glycol at $\mathrm{Ni}$ to propylene glycol ratio of $1: 100$. As seen from the electron microscopy images, the final product contains large crystals of the unreacted nickel compound of $\sim 1.5 \mu \mathrm{m}$ in width and $\sim 8 \mu \mathrm{m}$ in length and small (100-150 nm in size) spherical nickel nanoparticles nucleated directly on the crystals from which the nanowires are growing.

The synthesized complex $\mathrm{Ni}\left(\mathrm{N}_{2} \mathrm{H}_{4}\right)_{2}(\mathrm{CHOO})_{2}$ was further used as a starting material (precursor) for synthesis of nickel nanoparticles via the reduction route. In this synthetic procedure, a weighed amount of the precursor was mixed with ethylene glycol at $\mathrm{Ni}\left(\mathrm{N}_{2} \mathrm{H}_{4}\right)_{2}(\mathrm{CHOO})_{2}$ to ethylene glycol weight ratio of $1: 100$ and reduced by hydrazine hydrate at $130^{\circ} \mathrm{C}$. Electron microscopy images of the samples taken out from the reaction mixture at different reaction time periods are given in Figure 5. A SEM image of the initial compound $\mathrm{Ni}\left(\mathrm{N}_{2} \mathrm{H}_{4}\right)_{2}(\mathrm{CHOO})_{2}$ prior to starting the reduction process is given in Figure 1(a). As seen, the compound is present as highly elongated crystals of about 400 to $800 \mathrm{~nm}$ in width and about 4 microns in length. Figure 5 shows SEM and TEM images of the products obtained from a reduction time of 1 and $2 \mathrm{~min}$. In the figure, the early growth of nanowires can be seen. It is also seen that small spherical particles of 50$150 \mathrm{~nm}$ in size are formed on the initial crystal surface while 
anisotropic nanostructures are growing away from it. These small Ni nanocrystals may act as nucleation sites, allowing the newly reduced nickel atoms from the solution to be absorbed thereon, which results in the formation of the larger wire-like shaped particles. So the wires are likely formed via adatom addition and direct growth on the nickel seeds. Five minutes after the reaction started, the precursor has been completely reduced resulting in the formation of nickel nanowires with an average size of about $100 \mathrm{~nm}$ in diameter and 2-4 microns in length (Figures 4(a) and 4(b)). According to the XRD data, the product obtained was a pure metallic nickel with a crystallite size of $15 \mathrm{~nm}$.

There could be a few reasons why such a shape is realized during the reduction of nickel formate by hydrazine hydrate in ethylene glycol. The first reason could be an inherent selfgenerated magnetic field which nickel particles possess and due to which they can aggregate along the magnetic force lines forming the $1 \mathrm{D}$ nanostructures. According to information in the literature [39-41], nanoparticles with magnetic properties can spontaneously align into chain structures. Huelser et al. [42], using model calculations, showed that formation of the chains of iron nanoparticles up to $300 \mu \mathrm{m}$ in length can be explained by a magnetic interaction between the ferromagnetic particles in the reaction medium. Computational studies of purely metallic ( $\mathrm{Fe}, \mathrm{Co}$, and $\mathrm{Ni}$ ) magnetic nanoparticle chains [43] have also confirmed that. So the strong magnetic dipole interactions between nickel particles can make them align and could be the reason for the formation of the magnetic wire-like structures.

On the other hand, the solvent used for nanowires preparation is known to have a significant effect on their morphology. Some solvents which are able to interact with the metal atoms can promote the growth of anisotropic structures whereas others suppress completely such growth. The polyol medium we used in the synthesis may well be responsible for the formation of the wire-like structures acting as a capping agent thus affecting the nucleation and growth rates of the nickel crystals. As found by Gong et al. [44], the morphologies of the $\mathrm{Ni}$ nanocrystallites are closely related to the types of the solvents and only the solvents with double hydroxyl groups could be used for the preparation of the $\mathrm{Ni}$ fibres. So it is reasonable to suppose that ethylene glycol is able to influence the growth of anisotropic nanostructures via binding to certain $\mathrm{Ni}$ nanocrystal facets and that can result in the formation of wire-like structures. It should be noted that nickel nanowires are formed in the ethylene glycol solution but they do not form under the same reaction conditions when changing the type of polyol. Thus, it was shown that nickel nanoparticles resulting from the reduction of nickel formate in diethylene glycol, 1,2-propylene glycol, and 1,5pentanediol do not possess a wire-like shape (Figure 6). As seen from the TEM micrograph given in Figure 6, nickel nanoparticles prepared in 1,2-propylene glycol are mostly spherical in shape and have smaller sizes, less than $50 \mathrm{~nm}$, and this morphology did not change even when varying the reaction conditions (temperature and precursor concentration). So the polyol type affects both the shape and size of the resultant nanoparticles. The reason may be that all these solvents, being more viscous than ethylene glycol, affect the

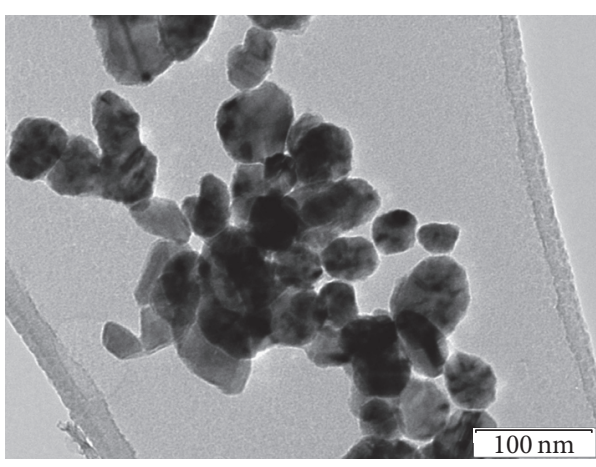

FIGURE 6: TEM image of nickel nanoparticles synthesized by reduction of nickel formate in 1,2-propylene glycol with hydrazine hydrate at $130^{\circ} \mathrm{C}$.

diffusion and growth processes, slowing them and inhibiting nanowire formation. As reported by Tao et al. [45], fast nucleation and fast growth promote nanowires formation so the ethylene glycol reaction medium seems to play an important role in the formation of nickel anisotropic nanostructures. Also it should be noted that polyols ability to tune particle size and shape is certainly related to their complexing strength (or their ability to coordinate the $\mathrm{Ni}$ (II) ions), which is not the same for different polyols and decreases when passing from a short chain polyol to a long chain polyol [46]. Therefore, the more stable intermediate formed in the Ni-EG system may be favourable to the nanowires formation but elucidation of this issue requires further investigation.

\section{Conclusions}

Nickel nanowires have been prepared in two simple ways: by reduction of both nickel formate and nickel hydrazine formate $\mathrm{Ni}\left(\mathrm{N}_{2} \mathrm{H}_{4}\right)_{2}(\mathrm{CHOO})_{2}$ with hydrazine hydrate in ethylene glycol at $130^{\circ} \mathrm{C}$ without the use of other components such as additional capping agents. The dependence of the structural characteristics of nickel nanoparticles on the synthesis conditions showed that an increase in the reaction time results in little change in the dimensions of the nanowires. An increase in the temperature results in an increase in the rate of the nickel reduction but does not change very much the dimensions and morphology of the resultant nanoparticles. A decrease in the nickel formate concentration in the solution results in a decrease in the diameters of the nanowires. Thus, at nickel to ethylene glycol ratios of $1: 20,1: 100,1: 400$, and 1:1000, the average diameters of the nanowires are $300,150,100$, and $50 \mathrm{~nm}$, respectively, while their lengths almost do not change, remaining in the range of $2-3 \mu \mathrm{m}$. Final morphology of the nickel nanoparticles was shown to depend on the choice of polyol used in the reaction. The mechanism of the nanowire formation has been discussed and it has been shown that, when using $\mathrm{Ni}\left(\mathrm{N}_{2} \mathrm{H}_{4}\right)_{2}(\mathrm{CHOO})_{2}$ as the precursor, $\mathrm{Ni}$ nanowires grow from spherical seeds initially formed on the crystal surface via heterogeneous nucleation. When using nickel formate, which is soluble in ethylene glycol, one could assume that $\mathrm{Ni}$ nanowires grow from spherical seeds initially formed in the solution via 
homogeneous nucleation. Although it might be possible that, due to the high temperature, which leads to higher reaction rates, large crystallites do not have time to be formed so the nanowires growth takes place on very small solid particles which appear in the solution just before the nickel reduction. The as-synthesized nickel nanowires are expected to show enhanced ferromagnetic properties and could be suited for use in catalysis.

\section{Competing Interests}

The authors declare that there is no conflict of interests regarding the publication of this paper.

\section{Acknowledgments}

This work was supported by the Russian Science Foundation, Research Project no. 15-13-00113.

\section{References}

[1] J. Hu, M. Ouyang, P. Yang, and C. M. Lieber, "Controlled growth and electrical properties of heterojunctions of carbon nanotubes and silicon nanowires," Nature, vol. 399, no. 6731, pp. 4851, 1999.

[2] C.-M. Liu, L. Guo, R.-M. Wang, Y. Deng, H.-B. Xu, and S. Yang, "Magnetic nanochains of metal formed by assembly of small nanoparticles," Chemical Communications, no. 23, pp. 27262727, 2004.

[3] W. Xu, K. Y. Liew, H. Liu, T. Huang, C. Sun, and Y. Zhao, "Microwave-assisted synthesis of nickel nanoparticles," Materials Letters, vol. 62, no. 17-18, pp. 2571-2573, 2008.

[4] J. S. Bradley, B. Tesche, W. Busser, M. Maase, and M. T. Reetz, "Surface spectroscopic study of the stabilization mechanism for shape- selectively synthesized nanostructured transition metal colloids," Journal of the American Chemical Society, vol. 122, no. 19, pp. 4631-4636, 2000.

[5] F. Ma, J. Ma, J. Huang, and J. Li, "The shape dependence of magnetic and microwave properties for Ni nanoparticles," Journal of Magnetism and Magnetic Materials, vol. 324, no. 2, pp. 205-209, 2012.

[6] J. Wang, L. Y. Zhang, P. Liu et al., "Preparation and growth mechanism of nickel nanowires under applied magnetic field," Nano-Micro Letters, vol. 2, no. 2, pp. 134-138, 2010.

[7] K. R. Krishnadas, P. R. Sajanlal, and T. Pradeep, "Pristine and hybrid nickel nanowires: template-, magnetic field-, and surfactant-free wet chemical synthesis and raman studies," Journal of Physical Chemistry C, vol. 115, no. 11, pp. 4483-4490, 2011.

[8] A. Cortés, G. Riveras, J. L. Palma et al., "Single-Crystal growth of nickel nanowires: influence of deposition conditions on structural and magnetic properties," Journal of Nanoscience and Nanotechnology, vol. 9, no. 3, pp. 1992-2000, 2009.

[9] Z. R. Smith, R. L. Smith, and S. D. Collins, "Mechanism of nanowire formation in metal assisted chemical etching," Electrochimica Acta, vol. 92, pp. 139-147, 2013.

[10] J. Zhang, W. Xiang, Y. Liu, M. Hu, and K. Zhao, "Synthesis of high-aspect-ratio nickel nanowires by dropping method," $\mathrm{Na}$ noscale Research Letters, vol. 11, no. 1, pp. 118-122, 2016.
[11] Z. Xia and W. Wen, "Synthesis of nickel nanowires with tunable characteristics," Nanomaterials, vol. 6, no. 1, article no. 19, 2016.

[12] W. Zhou, K. Zheng, L. He et al., "Ni/Ni3C core-shell nanochains and its magnetic properties: one-step synthesis at low temperature," Nano Letters, vol. 8, no. 4, pp. 1147-1152, 2008.

[13] H. Wu, R. Zhang, X. Liu, D. Lin, and W. Pan, "Electrospinning of Fe, Co, and Ni nanofibers: synthesis, assembly, and magnetic properties," Chemistry of Materials, vol. 19, no. 14, pp. 3506-3511, 2007.

[14] M. Jamal, M. Hasan, A. Mathewson, and K. M. Razeeb, "Disposable sensor based on enzyme-free Ni nanowire array electrode to detect glutamate," Biosensors and Bioelectronics, vol. 40, no. 1, pp. 213-218, 2013.

[15] L. He, Z.-M. Liao, H.-C. Wu et al., "Memory and threshold resistance switching in $\mathrm{Ni} / \mathrm{NiO}$ core-shell nanowires," Nano Letters, vol. 11, no. 11, pp. 4601-4606, 2011.

[16] S. Senapati, S. K. Srivastava, S. B. Singh, and H. N. Mishra, "Magnetic Ni/Ag core-shell nanostructure from prickly $\mathrm{Ni}$ nanowire precursor and its catalytic and antibacterial activity," Journal of Materials Chemistry, vol. 22, no. 14, pp. 6899-6906, 2012.

[17] N. Gao, H. Wang, and E.-H. Yang, "An experimental study on ferromagnetic nickel nanowires functionalized with antibodies for cell separation," Nanotechnology, vol. 21, no. 10, 2010.

[18] F. Byrne, A. Prina-Mello, A. Whelan et al., "High content analysis of the biocompatibility of nickel nanowires," Journal of Magnetism and Magnetic Materials, vol. 321, no. 10, pp. 1341$1345,2009$.

[19] S. Wen and J. Szpunar, "Direct electrodeposition of highly ordered magnetic nickel nanowires on silicon wafer," Micro \& Nano Letters, vol. 1, no. 2, pp. 89-93, 2006.

[20] A. K. Bentley, M. Farhoud, A. B. Ellis, G. C. Lisensky, A.-M. L. Nickel, and W. C. Crone, "Template synthesis and magnetic manipulation of nickel nanowires," Journal of Chemical Education, vol. 82, no. 5, pp. 765-768, 2005.

[21] P. Liu, Z. Li, B. Zhao, B. Yadian, and Y. Zhang, "Templatefree synthesis of nickel nanowires by magnetic field," Materials Letters, vol. 63, no. 20, pp. 1650-1652, 2009.

[22] H. Wang, X. Li, M. Li, K. Xie, and L. Liao, "Preparation of Ni/Cu composite nanowires," Beilstein Journal of Nanotechnology, vol. 6, no. 1, pp. 1268-1271, 2015.

[23] M. Li, K. N. Xie, J. W. Ye, Y. Z. Wu, and L. Li, "Facile synthesis of nickel nanowires under magnetic field," Materials Science and Technology, vol. 30, no. 6, pp. 712-714, 2014.

[24] Y. Soumare, A. Dakhlaoui-Omrani, F. Schoenstein, S. Mercone, G. Viau, and N. Jouini, "Nickel nanofibers and nanowires: elaboration by reduction in polyol medium assisted by external magnetic field," Solid State Communications, vol. 151, no. 4, pp. 284-288, 2011.

[25] H. Niu, Q. Chen, M. Ning, Y. Jia, and X. Wang, "Synthesis and one-dimensional self-assembly of acicular nickel nanocrystallites under magnetic fields," Journal of Physical Chemistry B, vol. 108, no. 13, pp. 3996-3999, 2004.

[26] N. Cordente, M. Respaud, F. Senocq, M.-J. Casanove, C. Amiens, and B. Chaudret, "Synthesis and magnetic properties of nickel nanorods," Nano Letters, vol. 1, no. 10, pp. 565-568, 2001.

[27] Z. P. Liu, S. Li, Y. Yang, S. Peng, Z. K. Hu, and Y. T. Qian, "Complex-surfactant-assisted hydrothermal route to ferromagnetic nickel nanobelts," Advanced Materials, vol. 15, no. 22, pp. 1946-1948, 2003. 
[28] H. M. Rietveld, "A profile refinement method for nuclear and magnetic structures," Journal of Applied Crystallography, vol. 2, pp. 65-71, 1969.

[29] F. Fievet, J. P. Lagier, B. Blin, B. Beaudoin, and M. Figlarz, "Homogeneous and heterogeneous nucleations in the polyol process for the preparation of micron and submicron size metal particles," Solid State Ionics, vol. 32-33, no. 1, pp. 198-205, 1989.

[30] D. Ai and S. Kang, "Synthesis of Ni nanopowders using an EHA system," Materials Transactions, vol. 47, no. 8, pp. 2056-2059, 2006.

[31] G. Viau, F. Fiévet-Vincent, and F. Fiévet, "Nucleation and growth of bimetallic CoNi and FeNi monodisperse particles prepared in polyols," Solid State Ionics, vol. 84, no. 3-4, pp. 259-270, 1996.

[32] P. B. Lond, P. S. Salmon, and D. C. Champeney, "Structure of $\mathrm{Ni}^{2+}$ solutions in ethylene glycol by neutron diffraction: an observed hydrogen bond between the solvent ligands in the first and second cation coordination shells?" Journal of the American Chemical Society, vol. 113, no. 17, pp. 6420-6425, 1991.

[33] S.-H. Wu and D.-H. Chen, "Synthesis and characterization of nickel nanoparticles by hydrazine reduction in ethylene glycol," Journal of Colloid and Interface Science, vol. 259, no. 2, pp. 282286, 2003.

[34] Y.-H. Choi, Y.-S. Chae, J.-H. Lee, Y.-W. Kwon, and Y.-S. Kim, "Mechanism of metal nanowire formation via the polyol process," Electronic Materials Letters, vol. 11, no. 5, pp. 735-740, 2015.

[35] C. Gong, J. Zhang, X. Zhang et al., "Strategy for ultrafine ni fibers and investigation of the electromagnetic characteristics," The Journal of Physical Chemistry C, vol. 114, no. 22, pp. 10101$10107,2010$.

[36] D. Nicholls and R. Swindells, "Hydrazine complexes of nickel(II) chloride," Journal of Inorganic and Nuclear Chemistry, vol. 30, no. 8, pp. 2211-2217, 1968.

[37] L. Guo, C. Liu, R. Wang, H. Xu, Z. Wu, and S. Yang, "Largescale synthesis of uniform nanotubes of a nickel complex by a solution chemical route," Journal of the American Chemical Society, vol. 126, no. 14, pp. 4530-4531, 2004.

[38] J. W. Park, E. H. Chae, S. H. Kim et al., "Preparation of fine Ni powders from nickel hydrazine complex," Materials Chemistry and Physics, vol. 97, no. 2-3, pp. 371-378, 2006.

[39] M. P. Pileni, "Nanocrystal self-assemblies: fabrication and collective properties," Journal of Physical Chemistry B, vol. 105, no. 17, pp. 3358-3371, 2001.

[40] M. Grzelczak, J. Vermant, E. M. Furst, and L. M. Liz-Marzán, "Directed self-assembly of nanoparticles," ACS Nano, vol. 4, no. 7, pp. 3591-3605, 2010.

[41] H. Kitching, M. J. Shiers, A. J. Kenyon, and I. P. Parkin, "Selfassembly of metallic nanoparticles into one dimensional arrays," Journal of Materials Chemistry A, vol. 1, no. 24, pp. 6985-6999, 2013.

[42] T. P. Huelser, H. Wiggers, P. Ifeacho, O. Dmitrieva, G. Dumpich, and A. Lorke, "Morphology, structure and electrical properties of iron nanochains," Nanotechnology, vol. 17, no. 13, pp. 3111-3115, 2006.

[43] A. Hucht, S. Buschmann, and P. Entel, "Molecular dynamics simulations of the dipolar-induced formation of magnetic nanochains and nanorings," EPL (Europhysics Letters), vol. 77, no. 5, Article ID 57003, 2007.

[44] C.-H. Gong, C. Q. Du, Y. Zhang, Z.-S. Wu, and Z.-J. Zhang, "Morphology-controlled synthesis of nickel nanostructures under magnetic fields," Chinese Journal of Inorganic Chemistry, vol. 25, no. 9, pp. 1569-1574, 2009.
[45] A. R. Tao, S. Habas, and P. Yang, "Shape control of colloidal metal nanocrystals," Small, vol. 4, no. 3, pp. 310-325, 2008.

[46] K. J. Carroll, J. U. Reveles, M. D. Shultz, S. N. Khanna, and E. E. Carpenter, "Preparation of elemental $\mathrm{Cu}$ and Ni nanoparticles by the polyol method: an experimental and theoretical approach," Journal of Physical Chemistry C, vol. 115, no. 6, pp. 2656-2664, 2011. 

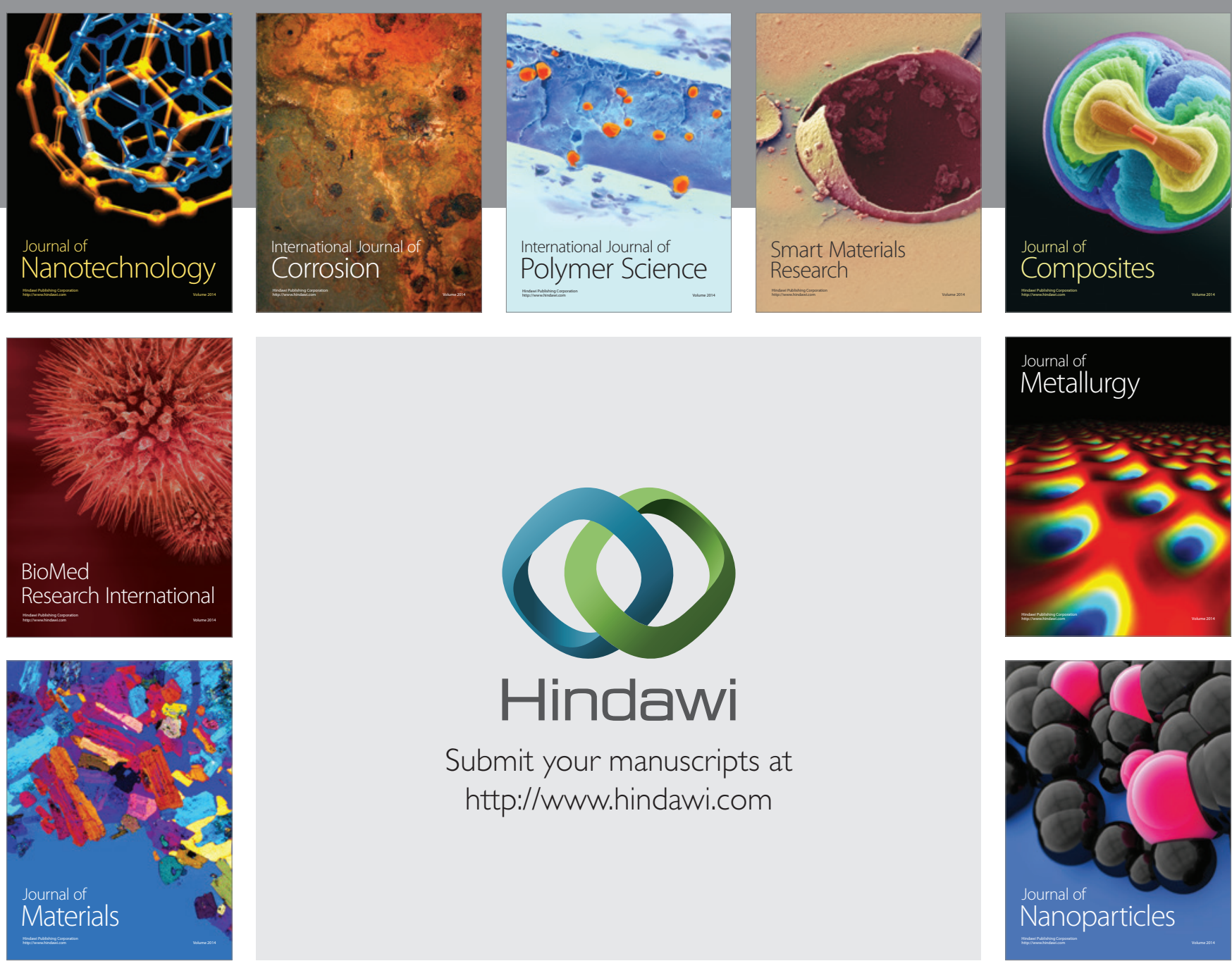

\section{Hindawi}

Submit your manuscripts at

http://www.hindawi.com

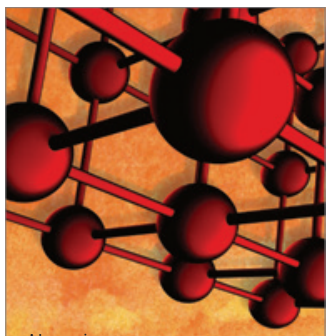

Materials Science and Engineering
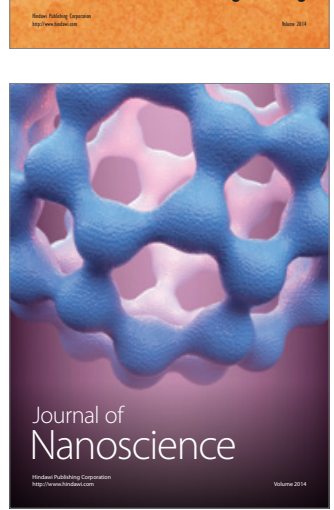
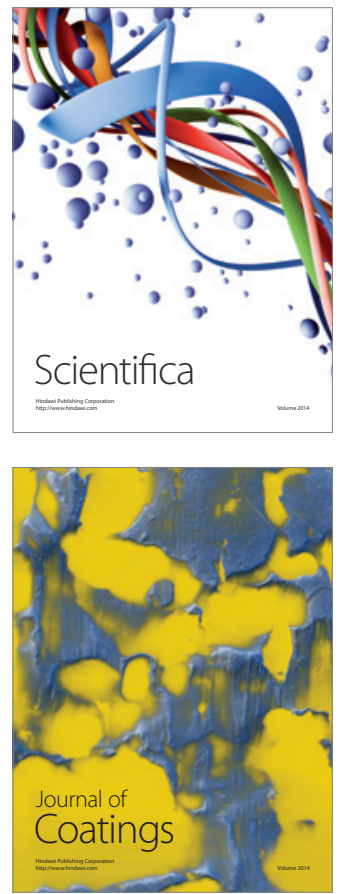
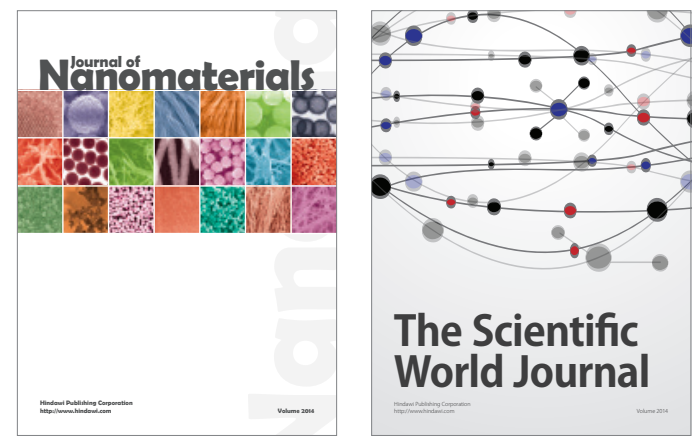

The Scientific World Journal
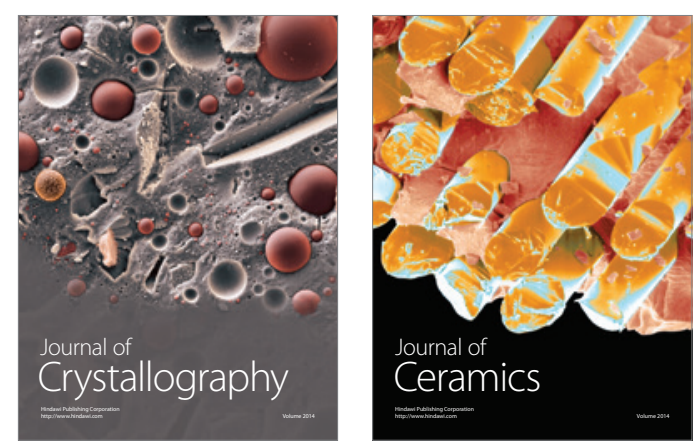
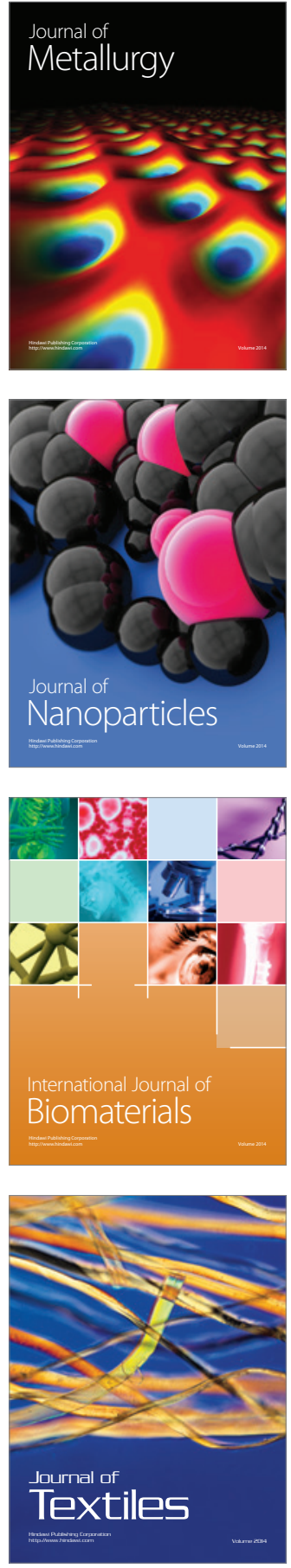